\title{
Location methods of oscillation sources in power systems: a survey
}

\author{
Bin WANG ${ }^{1}$, Kai SUN ${ }^{1}$ (D)
}

\begin{abstract}
The deployment of a synchrophasor-based widearea measurement system (WAMS) in a power grid largely improves the observability of power system dynamics and the operator's real-time situational awareness for potential stability issues. The WAMS in many power grids has successfully captured system oscillation events, e.g. poorly damped natural oscillations and forced oscillations, from time to time. To identify the root cause of an observed oscillation event for further mitigation actions, many methods have been proposed to locate the source of oscillation based on different ideas and principles. However, most methods proposed so far for locating the oscillation source in a power grid are not reliable enough for practical applications. This paper presents a comprehensive review of existing location methods, which basically fall into four major categories, plus a few other methods. Their advantages and disadvantages are discussed in detail. Some trends and challenges on the problem of oscillation source location are pointed out along with potential future research directions. Finally, a practical, general scheme for oscillation source location using available location methods is suggested and analyzed.
\end{abstract}

Crosscheck date: 24 March 2016

Received: 17 November 2015/Accepted: 20 April 2016/Published online: 27 July 2016

(C) The Author(s) 2016. This article is published with open access at Springerlink.com

$\triangle$ Kai SUN

kaisun@utk.edu

Bin WANG

bwang@utk.edu

1 University of Tennessee, Knoxville, TN 37996, USA
Keywords Power oscillation, Oscillation source location, Traveling wave, Damping torque, Mode shape, Transient energy, Oscillation test cases library

\section{Introduction}

Damped oscillations are a normal phenomenon for any disturbed system showing that the system is approaching back to its equilibrium. However, sustained oscillations could happen in reality where the possible causes include improper operating conditions, periodic disturbances or malfunctioning controllers. Such unexpected sustained oscillations may reduce the power transfer limit and even result in detrimental consequences on the system equipment. To solve this problem, researches on the analysis, detection, classification, location and control design have always been active during the past several decades while only a few of them have been integrated into system control centers to help system operators [1, 2].

Since sustained oscillations represent a risk for instability or insecurity of power systems, they should be mitigated as soon as possible. The location of the oscillation source is usually a prerequisite of the mitigation actions and the elimination of the source would always be the most straightforward and effective remedy. This paper presents a survey focusing on the methods for locating the oscillation source.

In the current literature, two types of mechanisms for sustained oscillations, i.e. poorly damped natural oscillations and forced oscillations, have been extensively investigated and explained for observed oscillation events, while some other mechanisms have also been discovered in analyses $[3,4]$ which are based either on Hopf bifurcation caused by slowly varying parameters or on practically 
impermissible nonlinear behavior of the system, e.g. outof-step condition. The discoveries and investigations of these mechanisms not only provide a better understanding of the oscillation phenomenon in power systems, but also are of fundamental importance for laying foundations for different location methods.

Usually, the oscillation source is implicitly defined as a physical device which causes oscillations following a certain mechanism. In practice, causes of sustained oscillations could be the excitation system [5], diesel engine [6], synchrotron as a cyclic load [7], control valve [8], turbopressure pulsation [9], governor control [10], asynchronous parallelizing of synchronous generators [11] and improper parameters for the steam turbine controller [12], et al. The purpose of a location method is to find the geographical location of the source, e.g. down to the substation level or control block level. This paper will cover the location methods down to the substation level, while the time localization [13] or device level localization [14-16] are not the focus of this paper.

Generally speaking, the basic requirements for location methods should include (1)-(3) below. In practice, besides the basic requirements, desired location methods should further include (4)-(9).

(1) A rigorous theoretical foundation

(2) The adaptability to different network topologies, different causes of oscillations and different models of dynamic elements that exist in reality

(3) The ability to reliably locate the source of oscillation without any false alarm

(4) Fully measurement-based, i.e. free of the system model

(5) Free of additional equipment or only requiring the minimum equipment to be installed that are inevitably necessary

(6) Able to provide useful information even with partial observability, i.e. when only partial system states are monitored

(7) Robustness against measurement noises and even missing data

(8) Computationally efficient

(9) Capable of working continuously in real time

However, many location methods in the current literature cannot even meet the above basic requirements. Thus, they are not ready for practical use.

This paper will summarize the principle, advantages and disadvantages of each reviewed method. Then, a discussion is provided on the trends and challenges to the location of oscillation source. Finally, a general scheme is suggested on how to utilize current available methods in practice and the conclusion is presented.

\section{Summary of the survey}

The oscillation source location problem has raised lots of attentions in the past decade and many papers on this topic have been published since 2010 , including 30 journal papers, 8 conference papers and 1 dissertation. These publications can be classified into four major categories plus a few other methods: 6 papers based on traveling wave, 2 papers based on damping torque, 3 papers based on mode shape estimation, 19 papers and 1 dissertation based on energy and 8 papers using other methods. Figure 1 shows how many papers published in each individual year and how many papers had been published by each year since 2010. Although this review only covers most related papers from IEEE Xplore and China National Knowledge Infrastructure (CNKI), it should be able to represent most types of methods for locating the oscillation source to the best of our knowledge.

\section{Traveling wave based methods}

Traveling wave based methods utilize the principle of the electromechanical wave propagation [17] to locate the oscillation source. Ideally, if the accurate detection of the arrival time of the oscillation at different locations and the actual wave speed map [18] were both available, the source location result should be correct and accurate. It is shown in [19] that the earliest detected peak of the oscillation always first appears at the generator with forced oscillation, i.e. oscillation source. Such phenomenon is claimed in another way [20] that the oscillation phase of the bus far away from the disturbance is lagging that of the bus close to the disturbance.

The similarity function is adopted in [21] for detecting the time differences of the arrival times among different generators connected to a common bus using terminal voltages, while the leading generator is identified as the oscillation source. To deal with large scale power systems, an additional device called voltage measuring unit (VMU) is introduced in $[22,23]$ to overcome the data quality issue

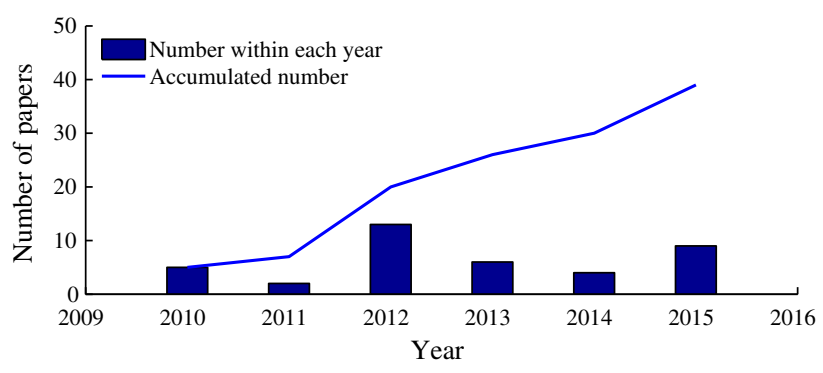

Fig. 1 Publications on oscillation source location 
of PMUs. With the assumption that the wave speeds throughout the network are roughly the same, the oscillation source is identified as the location associated with an abnormal wave speed. This method not only requires additional equipment to be installed at some designed locations, but also may possibly misjudge the source location when wave speeds vary significantly throughout the network or when the actual source is close to a certain VMU. A similar method in [24] utilizes the PMU data to locate the source, which employs the least mean square method to estimate the time differences of oscillation arrival times between different locations.

Traveling wave based methods only require measurements corresponding to the a few starting periods of the oscillation to determine the arrival time or time differences between different locations. The advantage of this category of methods is their fast response. Regardless the PMU data quality issue, to make these methods applicable in practice, the detection accuracy of arrival time, the impact from the non-constant wave speed throughout the network and the adaptability to different network topologies should be first investigated and guaranteed.

\section{Damping torque based methods}

In the damping torque based methods, the generator with a negative damping torque coefficient is identified as the oscillation source. The development of this category of methods depends on the extension of the damping torque concept from a single-machine-infinite-bus system (SMIB) to a multi-machine system.

The concept of damping torque was first introduced to offer a physical insight to system stability problem on an SMIB system [25]. For an SMIB system, the damping torque coefficient can be estimated using the system response by using the least square method [26, 27] or Kalman filter [28]. To extend the concept to multi-machine power systems, the first attempt in [29] estimates the damping torque for each single generator using its own electrical torque, rotor angle and rotor speed trajectories based on the least square error. However, this intuitive extension is found to be valid only when the speed deviations for all other generators are zeros, while it is not tenable in general [30]. Another extension of this concept is mentioned in [31], where the damping torque coefficient for the same generator is supposed to be different with respect to different oscillation modes such that it should be calculated separately for each mode. Thus, the damping torque of a generator in a multi-machine system could be estimated using the system trajectories based on a certain modal analysis method, like total least square-estimation of signal parameters via rotational invariance technique (TLSESPRIT) [31] or Prony [32].
The damping torque based location methods have clear physical meaning and could be applied locally for each individual generator, i.e. distributed implementation. Thus, this type of methods could be promising for locating the source which has bad damping. However, this category of methods may fail to work under some forced oscillation cases [33]. In addition, to estimate the damping torque from system trajectories, the measurements of generator electromagnetic torque, rotor angle and speed are often required, which, however, are not always directly measured by present PMUs.

\section{Mode shape estimation based methods}

Mode shape represents the relative magnitude and phasing of the oscillation throughout the system. It is defined based on the right eigenvectors of the state matrix of the linearized system model. However, the accurate system model is often difficult to obtain and the model based mode shape analysis has only been applied to test systems for a better understanding. To utilize the mode shape information in real systems, many measurementbased methods for estimating the mode shape have been proposed. An overview on existing estimation techniques using either ring-down signals or ambient signals can be found in [34] and its references.

This category of methods locates the oscillation source by estimating the mode shape using measurements from different locations and then identifies the source based on the mode shape. A phenomenon is discovered in [35] on a 2-machine example that the generator leading in mode shape phase contributes less damping. Then, it is intuitively concluded that the leading generator is the oscillation source. For large power systems, the leading generator in an identified leading group is identified as the oscillation source. Although this method is able to identify the oscillation source in many tested cases, it may possibly fail or provide wrong results [33] and the fundamental location principle has not been rigorously proved. Paper [36] compares the mode shape phasing difference between the deviations of mechanical power and electric power for each individual generator, and identifies the generator whose mechanical power is leading the electric power as the oscillation source.

The existing mode shape estimation based location methods always attempt to support their principles by finding a link to the damping concept. There is a lack of investigations on under what conditions the estimated mode shape from measurements conveys the information for locating the oscillation source. However, it was reported that under resonance condition the estimated mode shape from measurements could be quite different 
compared to the one from model-based eigenanalysis [37] and mode shape information may not help in locating the forced oscillation source [38].

\section{Energy based methods}

Transient energy function (TEF) is an application of Lyapunov function in power system stability analysis [37], which is usually defined as the sum of the kinetic and potential energies of all generators in the synchronous coordinate framework.

Papers [39] and [40] introduced the TEF based method for analyzing and characterizing the power system oscillations. Papers [41-43] extended the transient energy concept from generators to the branches in the network, which is called branch potential energy (BPE). The first attempt to apply the TEF or BPE to locate the source of forced oscillation is presented in [44] by monitoring the energy flow throughout the network and identifying the generator which injects the energy into the network as the source. Both of [45, 46] and [47] proved that the energy dissipation is consistent with the damping torque under some assumptions and the source can be identified as the component producing energy, which means a negative contribution to the damping. The energy function for generators based on the Port-Controlled Hamiltonian theory is established in [48] which can consider the excitation system and governor models. Then, a location method based on this energy is proposed in [49] by monitoring the energy injected to the network at each port and identifying the port with positive energy injection as the source. However, it is discovered that the location result from the energy based methods may be significantly affected by the load model [50], or even misleading [51]. Another phenomenon called the concentric relaxation is introduced in [51] for power systems under forced oscillations, which could help avoid the erroneous location results. To date, this category of methods has been largely applied to power systems in the past five years [52-63].

The energy based methods are capable of identifying generator-type oscillation source since the energy dissipation is proved to be consistent with the damping toque. When applying the energy based methods to monitor the energy flows on branches in the network, the directions of the energy flows may be significantly affected by nearby loads such that the results may be misjudged. However, how to utilize the characteristics of the oscillations, such as the concentric relaxation phenomenon for forced oscillation, to accurately and reliably locate the source still needs more investigations.

\section{Other methods}

Besides the above methods, several other methods are briefly summarized in this section. The equivalent circuit based method in 7.1 is derived from the energy method, which provides another representation of the system under oscillations. The rest of the methods in 7.2-7.5 either depend on the system model or cannot provide a clear location of the oscillation source, which makes them less practical.

\subsection{Equivalent circuit based method}

An "equivalent circuit" representation for power systems under oscillations is proposed in [64, 65], which adopts the phasor concept for the deviations of power and frequency, and the "source" in the "circuit" is identified as the source of the oscillation.

\subsection{Hybrid simulation based method}

A hybrid dynamic simulation method is proposed in $[66,67]$ to locate the oscillation source, which is based on both the system model and the measurement data. The location idea is to first replace part of the differential and algebraic variables, say $V_{p}$, with known measurements and perform the time domain simulation for the rest of the variables, say $V_{r}$. If the difference between the simulated response and the corresponding measurement data for $V_{r}$ is larger than a pre-designed threshold, the source is identified to be within the area associated with $V_{r}$. Repeating the process will provide a more accurate location for the source.

\subsection{Artificial intelligence (AI) based method}

An AI based method is proposed in [68] to locate the source of forced oscillation. Based on different operating conditions, the Characteristic ELLipsoid (CELL) is used to offline train a decision tree considering the sources respectively added to different generators or loads. Then, monitoring the parameters of the CELL using PMU measurements can help locate the source of the forced oscillation.

\subsection{Graph-theoretic based method}

A graph-theoretic and measurement-based method is proposed in [69] for locating the input disturbance in large networked dynamic systems. This method first applies a system identification routine to construct the input-output transfer matrix and calculate the array of nominal localization keys. Then, an estimated localization key from local 
PMU measurements can be obtained for the corresponding local area. Comparing the estimated key with the nominal keys will indicate the location of the source.

\subsection{Generalized linear model based method}

This location method is reported in [70, 71]. Firstly, a generalized linear model for the system is fitted using the measurement data. Then, such fitted model may help determine variables that have significant effects on mode damping, which might be related to the oscillation source.

\section{Discussions and conclusion}

A summary of reviewed location methods is provided in Table 1. It can be seen that there is a significant gap between the current available methods in the literature and the desired location method mentioned in the introduction section. This section will point out some critical trends and challenges to the development of the oscillation source location problem and then provide a general scheme on how to make use of current available methods in practice.

Table 1 Summary of reviewed location methods

\begin{tabular}{|c|c|c|c|c|}
\hline Category & Key idea & Advantages & Disadvantages & References \\
\hline $\begin{array}{l}\text { Traveling } \\
\text { wave }\end{array}$ & $\begin{array}{l}\text { The closer to the source, the earlier the location } \\
\text { will exhibit oscillations }\end{array}$ & Fast & $\begin{array}{l}\text { Inaccurate and unreliable detection of } \\
\text { oscillation arrival time. Unavailability } \\
\text { of the wave speed map in real-time. A } \\
\text { lack of investigations for multi-mode } \\
\text { oscillation cases. }\end{array}$ & [19-24] \\
\hline $\begin{array}{l}\text { Damping } \\
\text { torque }\end{array}$ & $\begin{array}{l}\text { The generator with a negative damping torque } \\
\text { coefficient is the source }\end{array}$ & $\begin{array}{l}\text { Clear physical } \\
\text { meaning; allows } \\
\text { for distributed } \\
\text { implementation }\end{array}$ & $\begin{array}{l}\text { Possible unavailability of rotor angle and } \\
\text { speed data. Possible failure under forced } \\
\text { oscillation cases. }\end{array}$ & {$[31,32]$} \\
\hline Mode shape & $\begin{array}{l}\text { Largest magnitude, most leading phase of the } \\
\text { mode shape or their combinations may } \\
\text { indicate the source }\end{array}$ & $\begin{array}{l}\text { Can deal with multi- } \\
\text { mode oscillations }\end{array}$ & $\begin{array}{l}\text { A lack of theoretical foundation. Possible } \\
\text { failure for weakly damped/undamped } \\
\text { oscillation and forced oscillation cases. }\end{array}$ & {$[35-38]$} \\
\hline Energy & $\begin{array}{l}\text { The device producing dissipation energy is the } \\
\text { source }\end{array}$ & $\begin{array}{l}\text { Allows for } \\
\text { distributed } \\
\text { implementation }\end{array}$ & $\begin{array}{l}\text { Too strong assumptions used for modeling } \\
\text { loads and the network. }\end{array}$ & [44-63] \\
\hline $\begin{array}{l}\text { Equivalent } \\
\text { circuit }\end{array}$ & $\begin{array}{l}\text { The source of the equivalent circuit is the } \\
\text { source of the oscillation }\end{array}$ & $\begin{array}{l}\text { Could be linked to } \\
\text { energy based } \\
\text { method }\end{array}$ & $\begin{array}{l}\text { Possible failure when phasor concept } \\
\text { cannot be applied, e.g. non-sinusoidal } \\
\text { oscillations. A lack of theoretical } \\
\text { investigation for multi-mode oscillation } \\
\text { cases. }\end{array}$ & {$[64,65]$} \\
\hline Hybrid & $\begin{array}{l}\text { A larger difference between model-based } \\
\text { simulations and measurements indicates the } \\
\text { source }\end{array}$ & $\begin{array}{l}\text { Not limited to the } \\
\text { level of the model } \\
\text { detail }\end{array}$ & $\begin{array}{l}\text { Possible unavailability of accurate model } \\
\text { of the entire system. }\end{array}$ & {$[66,67]$} \\
\hline AI & $\begin{array}{l}\text { An offline trained decision tree from model- } \\
\text { based simulations may use online } \\
\text { measurements to locate the source }\end{array}$ & Fast & $\begin{array}{l}\text { Possible unavailability of accurate model } \\
\text { of the entire system. Can be only } \\
\text { applied to forced oscillation cases. }\end{array}$ & {$[68]$} \\
\hline $\begin{array}{l}\text { Graph } \\
\text { theoretic } \\
\text { method }\end{array}$ & $\begin{array}{l}\text { Comparing the locally estimated key (from } \\
\text { local measurements) and the centralized } \\
\text { nominal keys (from model) gives the source. }\end{array}$ & $\begin{array}{l}\text { Distributed } \\
\text { implementation }\end{array}$ & $\begin{array}{l}\text { Model-based partition of the whole system } \\
\text { into areas is required and the source is } \\
\text { only located down to the area level. }\end{array}$ & {$[69]$} \\
\hline $\begin{array}{l}\text { Generalized } \\
\text { linear } \\
\text { model }\end{array}$ & $\begin{array}{l}\text { Based on an identified generalized linear } \\
\text { model, some significant variables having } \\
\text { contributions to the mode dynamics may be } \\
\text { related to the source }\end{array}$ & $\begin{array}{l}\text { Can find the state } \\
\text { variables related } \\
\text { to the source }\end{array}$ & No source location is clearly indicated & {$[70,71]$} \\
\hline
\end{tabular}




\subsection{Trends and challenges}

1) Definition and characterization of the source

Although lots of researchers and technical papers tried to address the oscillation source location problem, a clear mathematical definition and characterization of the oscillation source are still missing.

The difficulty in the definition may be due to the various or unknown causes of oscillations such that different sources may possibly have different mathematical models. However, investigations in this direction play an important role, which could be the common foundation for all location methods even based on different principles.

The intrinsic characterizations of the oscillation source have not been paid enough attentions to so far. Different methods use different criteria to characterize their sources. For instance, damping torque based methods characterize the source by the generator with a negative damping torque coefficient, and the energy based methods characterize the source as the generator producing dissipating energy. However, we cannot prove that the generator having positive damping torque coefficient will never be the oscillation source in any way, so the damping torque based methods may possibly give wrong location results [33]. A similar conclusion holds for other methods.

Thus, future research should include the rigorous definition of the oscillation source and its accurate characterization along with the conditions under which the definition and characterization are valid.

As most studies do, the linear analysis is usually adopted to analyze the sustained oscillations and in forced oscillation cases the injection signal is usually assumed to follow the sinusoidal waveform. However, these might not always be true. In reality, nonlinearity may possibly be presented. In the forced oscillation cases, the injection signal may possibly follow certain non-sinusoidal waveforms, e.g. rectangular or sawtooth, which should have different characteristics from electromechanical oscillation [72, 73]. Thus, future research topics should address these issues.

2) Need for measurement-based methods

Traditional model-based analyses cannot help much on the location problem until (i) the system dynamic model is accurate enough and (ii) the unknown source is also accurately modeled. In reality, the location and nature of the source are typically unknown and cannot be modeled before located, so promising location methods for practical applications are supposed to be measurement-based. Model-based analysis is still useful for validating the mechanism of an oscillation event and the principle of a location method.

However, before applying any measurement-based method to real systems, all issues about the acquisition of measurements should be considered in future research to check the performance of the method. Noise in the measurements should always be considered. Besides, if a measurement-based method is implemented in a centralized way, then issues from the communication such as the loss of synchronization of data and missing data should be considered. For methods designed for online applications, time delay should also be considered.

In addition, the capability of distributed implementation of a measurement-based method is also desired and should be a direction of future research. This capability will make the method to be free of communication issues.

In today's network, the measurement devices are usually not enough to reach a full observability of the entire system states. Thus, it is desired that a measurement-based method can also provide useful information based on measurements with only partial observability.

3) Capability assessment of location methods

Almost every location method was only tested with a few specifically designed cases to demonstrate its effectiveness when proposed. It could still be possible that the method may fail for some other cases, which are not included in the published papers.

It is difficult to guarantee that all possible situations are tested when evaluating a location method. However, it is always feasible to test all imaginable situations. To this end, the development of a test cases library is a need. A library for this purpose was proposed in [33], which contains 23 oscillation cases including 14 forced oscillation cases and 9 natural oscillation cases. Although many situations have been included in that library, other situations may probably exist and should be added in future, e.g. sustained oscillation cases with constantly changing system operating conditions, and forced oscillation cases where the external force is added to other signals in the excitation system or added to other control devices. In addition, this library only has generators represented by the classical model or round rotor model and all loads are in constant power model. Although most models there are representative, they can still be different from real systems. Therefore, any devices that exist in real systems and may cause oscillations, e.g. HVDC, wind turbine and other FACTs devices, should be considered to create additional cases to supplement the library in future.

\subsection{A general scheme for practical applications}

To give a possible solution to the oscillation source location problem faced by today's system operators and analysts, the following provides a general scheme using all available location methods and the latest test cases library. Other schemes can be created similarly by using other criteria to categorize the available location methods. 
Generally, by testing all cases in the test cases library, any location method can always be categorized into one of the three types below:

(1) Type-1: the methods whose location results are always correct but there could possibly be no location result found by the methods.

(2) Type-2: the methods that may provide multiple location results while the actual source is always included.

(3) Type-3: the methods whose location results may possibly miss the actual source and give wrong results.

This paper does not provide the categorization result of the reviewed methods, since there could be different configurations even for the same location method which will lead to different location performances.

After categorizing the location methods according to the above three types, system operators and analysts can make use of the Type- 1 and Type- 2 methods to locate the source or a few candidates of the source. Note that if all available methods fall into Type-3, then the oscillation source location problem cannot be solved by today's techniques even for ideally simulated cases. In such a case, there is no confident way that the system operators can follow to tackle the problem. They may rely on their experience or certain existing guidelines, or find some hints from the location results of Type- 3 methods.

When a sustained oscillation event happens and one or several candidates of the source are found by the Type- 1 and Type- 2 methods, verification procedures should be enabled for each of the source candidates. The development of the verification procedures are also a future research topic, which may include but not limited to: (i) recheck the presence of the oscillation after eliminating a certain source candidate; (ii) use internal measurements of a certain source candidate, if available, to find the possible cause of the oscillation, e.g. detailed field investigations in a suspicious plant or substation. If the actual cause can be found and it is consistent with the location result, then the source is correctly located. Otherwise, the oscillation event under study is not included in the library. In this situation, additional cases should be designed and added to the library to represent such oscillation event. Finally, all Type- 1 and Type- 2 location methods should re-categorized by the updated library for future use.

With Type- 1 and Type- 2 location methods and the test cases library, the above scheme for locating the oscillation source in practice should work in an iterative way: new location methods can be added when available and the test cases library can be updated by adding more cases that were not included.

\subsection{Conclusion}

This paper summarizes the methods for locating the oscillation source in power systems. The advantages and disadvantages of each method are analyzed. Some development trends and challenges are pointed out, including a few future research topics. A general scheme for locating the source of oscillation in practice is provided and analyzed.

To conclude, more efforts on the investigations and developments of oscillation source location methods will be constantly needed.

Acknowledgment This work was supported by the NSF CURENT Engineering Research Center (No. EEC-1041877).

Open Access This article is distributed under the terms of the Creative Commons Attribution 4.0 International License (http:// creativecommons.org/licenses/by/4.0/), which permits unrestricted use, distribution, and reproduction in any medium, provided you give appropriate credit to the original author(s) and the source, provide a link to the Creative Commons license, and indicate if changes were made.

\section{References}

[1] Zhang Q, Luo X, Litvinov E et al (2014) Advanced grid event analysis at ISO New England using PhasorPoint. In: IEEE PES General Meeting I Conference \& Exposition, National Harbor, MD, USA, 27-31 Jul 2014, 5 pp

[2] Zhou N, Dagle J (2015) Initial results in using a self-coherence method for detecting sustained oscillations. IEEE Trans Power Syst 30(1):522-530

[3] Deng J, Liu G, Bian E (1997) Study on Hopf bifurcation in low frequency oscillation. Proc CSEE 17(6):391-398

[4] Wang G, Shen C, Fu L et al (2015) Transformed periodic orbit mechanism of low frequency oscillations in power systems. J Circ Theor Appl, Int. doi:10.1002/cta.2073

[5] Demello F, Concordia C (1969) Concepts of synchronous machine stability as affected by excitation control. IEEE Trans Power App Syst 4:316-329

[6] Cournas C, Krassas N, Papadias B (1991) Analysis of forced oscillations in a multimachine power system. In: International Conference on Control, Edinburgh, UK, 25-28 Mar 1991, 6 pp

[7] Rostamkolai N, Piwko R, Matusik A (1994) Evaluation of the impact of a large cyclic load on the LILCO power system using time simulation and frequency domain techniques. IEEE Trans Power Syst 9(3):1411-1416

[8] Xu Y, He R, Han Z (2007) The cause analysis of turbine power disturbance inducing power system low frequency oscillation of resonance mechanism. Proc CSEE 27(17):84-87

[9] Xu Y, He R, Han Z (2008) Study on resonance mechanism of power system low frequency oscillation induced by turbo-pressure pulsation. Proc CSEE 28(1):47-51

[10] Zhu W, Zhou Y, Tan X et al (2009) Mechanism analysis of resonance-type low-frequency oscillation caused by networks side disturbance. Proc CSEE 29(25):37-42

[11] Yang D, Ding J, Li J et al (2011) Analysis of power system forced oscillation caused by asynchronous parallelizing of synchronous generators. Autom Electr Power Syst 35(10):99-103 
[12] Xu YH, Wang ZZ, Weng HJ (2013) A low frequency oscillation event caused by primal frequency modulation test and its mechanism analysis. Autom Electr Power Syst 37(23):119-124. doi:10.7500/AEPS20130621015

[13] Follum J, Pierre JW (2013) Initial results in the detection and estimation of forced oscillations in power systems. NAPS, Manhattan, KS 22-24:6 pp

[14] Li Y, Shen C, Liu F (2012) Oscillation source location in control devices of generators based on Hamiltonian realization. Autom Electr Power Syst 36(23):6-11

[15] Pu J, Tang Y, Shi X et al (2013) Oscillation source location based on abnormal signal analysis. In: IEEE PES APPEEC, Kowloon, Hong Kong, China, 8-11 Dec 2013, 5 pp

[16] Li W, Li Y, Zhou X et al (2013) Power system oscillation analysis and oscillation source location based on WAMS part 2: method of torques decomposition. Proc CSEE 33(25):47-53

[17] Thorp JS, Seyler CE, Phadke AG (1998) Electromechanical wave propagation in large electric power systems. IEEE Trans Circuits Syst I, Fundam Theory Appl 45(6):614-622

[18] Markham PN, Liu Y (2014) Electromechanical speed map development using FNET/GridEye frequency measurements. In: IEEE PES General Meeting I Conference \& Exposition, National Harbor, MD, USA, 27-31 Jul 2014, 5 pp

[19] Hu N, Li X, Yang Y et al (2013) Research for space-time variational features of frequency for power system forced oscillations and disturbance source location. J Sichuan Univ (Eng Sci Edn) 45(6):135-142

[20] Geng T, Zhang Z, Xiang L et al (2015) A locating and splitting scheme for disturbance source of forced power oscillation based on the propagation characteristic. Power Syst Protect Control 43(6):98-103

[21] Dong Q, Zhang L, Yan X (2012) Automatic location of generation units behaving as disturbance source of low-frequency oscillation. Power Syst Technol 36(10):265-269

[22] Dong Q, Liang J, Yan X, Yang R (2012) Locating method of disturbance source of low frequency oscillation in large scale power grid. Proc CSEE 32(1):78-83

[23] Dong Q, Zhang L, Yan X, Liu X (2012) Automatic locating source method of compelled resonance low frequency oscillation in power grid. Proc CSEE 32(28):68-75

[24] Zhu ZS, Liu DC, Liao QF et al (2015) Disturbance source location of forced power oscillation based on LMS time delay estimation method. Autom Electr Power Syst 39(4):58-62. doi:10.7500/AEPS20140729006

[25] DeMello F, Concordia C (1969) Concepts of synchronous machine stability as affected by excitation control. IEEE Trans Power App Syst 88(4):316-329

[26] Alden RTH, Shaltout AA (1979) Analysis of damping and synchronizing torques part I - a general calculation method. IEEE Trans Power App Syst 98(6):1696-1700

[27] Alden RTH, Shaltout AA (1979) Analysis of damping and synchronizing torques part II - effect of operating conditions and machine parameters. IEEE Trans Power App Syst 98(6): 1701-1708

[28] Feilat E A, Younan N (1999) Online adaptive assessment of the synchronizing and damping torque coefficients using Kalman filtering. In: Proceedings of the IEEE Southeastcon '99, Lexington, KY, USA, 4 pp

[29] Shaltout AA, Feilat EA (1992) Damping and synchronizing torque computation in multimachine power systems. IEEE Trans Power Syst 7(1):280-286

[30] Zheng X (2000) The complex torque coefficient approach's applicability analysis and its realization by time domain simulation. Proc CSEE 20(6):1-4
[31] Li Y, Huang Y, Liu J et al (2015) Power system oscillation source location based on damping toruqe analysis. Power Syst Protect Control 43(14):84-91

[32] Gao Y, Liu D, Huang G, Shi Q (2012) Locating method of disturbance source of forced power oscillation based on Prony analysis. CICED, Shanghai, pp 10-14

[33] Maslennikov S, Wang B, Zhang Q, Ma F et al (2016) A test cases library for methods locating the sources of sustained oscillations. 2016 IEEE PES General Meeting, Boston, MA, July $17-21$

[34] Dosiek L, Zhou N, Pierre JW et al (2013) Mode shape estimation algorithms under ambient conditions: a comparative review. IEEE Trans Power Syst 28(2):779-787

[35] Ashwal N, Wilson D, Parashar M (2014) Identifying sources of oscillations using wide area measurements. Grid of the Future Symposium, CIGRE US National Committee

[36] Wang M, Sun H (2014) An analysis method for forced power oscillation source detection. Proc CSEE 34(34):6209-6215

[37] Myers R Trudnowski D (2013) Effects of forced oscillations on spectral-based mode-shape estimation. IEEE PES GM, Vancouver, BC, Canada, pp. 21-25

[38] Sarmadi S, Venkatasubramanian V (2015) Inter-area resonance in power systems from forced oscillations. IEEE Trans Power Syst 99:1-9

[39] Jinag C, McCalley J, Kommareddy M (1996) An energy approach to analysis of interarea oscillations in power systems. IEEE Trans Power Syst 11(2):734-740

[40] Messina A, Ochoa M, Barocio E (2001) Use of energy and power concepts in the analysis of the inter-area mode phenomenon. Electr Power Syst Res 59(2001):111-119

[41] Moon Y, Cho B, Lee Y, Kook H (1999) Derivation of energy conservation law by complex line integral for the direct energy method of power system stability. In: Proceedings of the $38^{\text {th }}$ IEEE Conference on Decision and Control, Phoenix, AZ, USA, 7-10 Dec, 1999, 6 pp

[42] Cai G, Mu G, Liu Z, Lin Z (1999) Network transient energy function defined on output trajectory. Autom Electr Power Syst 23(9):28-32

[43] Cai G, Mu G, Chan K, Lu F (2004) Branch potential energy method for power system transient stability assessment based on network dynamic variables. Proc CSEE 24(5):1-6

[44] Yu Y, Min Y, Chen L, Zhang Y (2010) Disturbance source location of forced power oscillation using energy functions. Autom Electr Power Syst 34(5):1-6

[45] Chen L, Min Y, Hu W (2012) Low frequency oscillation analysis and oscillation source location based on oscillation energy part one mathematical foundation and energy flow computation. Autom Electr Power Syst 36(3):22-27

[46] Chen L, Chen Y, Min Y et al (2012) Low frequency oscillation analysis and oscillation source location based on oscillation energy part two method for oscillation source location and case studies. Autom Electr Power Syst 36(4):1-5

[47] Chen L, Min Y, Hu W (2013) An energy-based method for location of power system oscillation source. IEEE Trans Power Syst 28(2):828-836

[48] Li Y, Shen C, Liu F (2012) An energy-based methodology for locating the source of forced oscillations in power systems. IEEE POWERCON, Auchland, New Zealand, 30 Oct-2 Nov, 2012, 6 pp

[49] Li Y, Shen C, Liu F (2013) A methodology for power system oscillation analysis based on energy structure. Autom Electr Power Syst 37(13):49-56. doi:10.7500/AEPS201211185

[50] Duan Q, Zhu Y, Zhou H, Ma J (2012) Impact of load model on disturbance sources locating based on energy function. Shaanxi Electr Power 8:16-19 
[51] Yang Y, Liu T, Li X et al (2013) An analysis to the concentric relaxation phenomenon of power system forced oscillations. J Sichuan Univ (Eng Sci Edn) 45(4):163-170

[52] Hu W, Lin T, Gao Y et al (2011) Disturbance source location of forced power oscillation in regional power grid. IEEE PEAM, Wuhan, China, 8-9 Sept 2011, 4 pp

[53] Yu Y, Min Y, Chen L, Ju P (2011) The disturbance source identification of forced power oscillation caused by continuous cyclical load. 4th International Conference on Electric Utility Deregulation and Restructuring and Power Technologies (DRPT), Weihai, Shandong, China, 6-9 July, 2011, 6 pp

[54] Yang D, Ding J, Li J et al (2012) A disturbance source location method for forced power oscillations based on parameter identification. Autom Electr Power Syst 36(2):26-30

[55] Hu W, Lin T, Gao Y et al (2012) Disturbance source location of forced power oscillation in regional power grid based on dissipation power. High Volta Eng 38(4):1006-1011

[56] Dong C, Liu D, Liao Q et al (2012) Research on low frequency oscillation in power grid and location of disturbance source based on energy function. Power Syst Technol 36(8):175-181

[57] Li W, Guo J, Li Y et al (2013) Power system oscillation analysis and oscillation source location based on WAMS part 1: method of cutset energy. Proc CSEE 33(25):41-46

[58] Wang N, Liao Q, Tang F, Li W (2013) Disturbance source identification based on cutset energy and sensitivity for forced power oscillation. Electr Power Autom Equip 33(1):75-80

[59] Chu X, Yin Y, Gao L et al (2014) A new forced oscillation disturbance source location method based on empirical mode theory. Proc CSEE 34(28):4906-4912

[60] Cao X, Liu T, Li X (2014) Disturbance source location for forced power oscillations by wind turbines. Electr Mach Control $18(10): 81-86$

[61] Tang F, Wang B, Liao Q et al (2015) Research on forced oscillations disturbance source locating through an energy approach. Int Trans Electr Energ Syst. doi:10.1002/etep.2080

[62] Shang X, Gu X, Liang H et al (2015) Location identification method of disturbance source in forced power oscillation considering unobservability of PMU information. Autom Electr Power Syst 39(10):56-62. doi:10.7500/AEPS20140515004

[63] Yu Y (2010) Study on low frequency oscillation in power systems based on energy methods. Dissertation submitted to Tsinghua University, Beijing, China, $138 \mathrm{pp}$

[64] Yang Y, Liu T, Li X et al (2012) An equivalent circuit approach to locate souce of power system forced power oscillation. Proc CSEE 36(11):101-108

[65] Yang YQ, Liu TQ, Li XY et al (2015) Discussion on energy mechanism of negative damping and oscillation source locating method based on equivalent circuit method. Autom Electr Power Syst 39(10):63-68. doi:10.7500/AEPS20140409012
[66] Ma J, Zhang P, Fu H et al (2010) Application of phasor measurement unit on locating disturbance source for low-frequency oscillation. IEEE Trans Smart Grid 1(3):340-346

[67] Wu H, Duan Q, Ma J (2012) Disturbance source self-diagnosis of the smart grid. Spring Congress on Engineering and Technology, Xi'an, China, 27-30 May, 2012, 4 pp

[68] Jiang C, Liu J, Liu Y et al (2015) Online forced oscillation detection and identification based on wide area measurement system and CELL theory. Electr Power Autom Equip 35(2):125-132

[69] Nudell T, Chakrabrtty A (2015) Graph-theoretic methods for measurement-based input localization in large networked dynamic systems. IEEE Trans Autom Control 60(8):2114-2128

[70] McNabb P, Bochkina N, Bialek J (2010) Oscillation source location in power systems using logic regression. IEEE PES ISGT Europe, Gothenburg, Sweden, 11-13 Oct 2010, 8 pp

[71] McNabb P, Bochkina N, Wilson D, Bialek J (2010) Oscillation source location using wavelet transforms and generalized linear models. In: IEEE PES Transmission and Distribution Conference and Exposition, New Orleans, LA, USA, 19-22 Apr 2010, $9 \mathrm{pp}$

[72] Wang B, Sun K et al (2014) A Study on Fluctuations in Electromechanical Oscillation Frequencies of Power Systems, IEEE PES General Meeting, National Harbor, MD

[73] Wang B, Sun K (2016) Formulation and Characterization of Power System Electromechanical Oscillations, IEEE Transactions on Power Systems (in press). doi:10.1109/TPWRS.2016. 2535384

Bin WANG received the B. S. and M.S. degrees in electrical engineering from Xi' an Jiaotong University, China, in 2011 and 2013, respectively. $\mathrm{He}$ is currently pursuing the $\mathrm{PhD}$ degree at the Department of EECS, University of Tennessee in Knoxville. His research interests include power system nonlinear dynamics, stability and control.

Kai SUN received the B.S. degree in automation in 1999 and the $\mathrm{PhD}$ degree in control science and engineering in 2004 both from Tsinghua University, Beijing, China. He is currently an assistant professor at the Department of EECS, University of Tennessee in Knoxville. He was a project manager in grid operations and planning at the EPRI, Palo Alto, CA from 2007 to 2012. His research interests include power system dynamics, stability and control and complex systems. Dr. Sun is an editor of the IEEE Transactions on Smart Grid and an associate editor of IET Generation, Transmission and Distribution. 